\title{
Multi-Pore Sensing of Virus Capsids in In-Plane Nanofluidic Devices.
}

\author{
Zachary D. Harms ${ }^{1}$, Daniel G. Haywood ${ }^{1}$, Lisa Selzer ${ }^{2}$, Andrew R. Kneller ${ }^{1}$, Adam Zlotnick ${ }^{2}$ and \\ Stephen C. Jacobson ${ }^{1}$ \\ 1. Department of Chemistry, Indiana University, Bloomington, IN, USA. \\ 2. Department of Molecular and Cellular Biochemistry, Indiana University, Bloomington, IN, USA.
}

The study of biomolecular complexes in the megaDalton size regime presents many analytical challenges. To understand biological processes at these length scales, we want to sense with single particle resolution, in solution, at relevant particle concentration, and at millisecond time scales. Nanopore sensors have these advantages and are well suited to study biological particles, such as virus capsids [1]. Paramount to creating a successful sensor is to understand particle interactions at the nanoscale. Our fabrication method allows fine control over sensing geometry and, thus, permits elucidation of the forces involved in particle transport. We report planar glass nanopores integrated into glass microfluidic devices to perform resistive-pulse sensing of hepatitis B virus (HBV) capsids. Multiple nanopores in series along the axis of the nanochannel probe single particles multiple times, to maximize the amount of information obtained from the experiment.

To fabricate the devices, V-shaped microchannels are first created in a glass substrate by UV photolithography and chemical etching. A focused ion beam (FIB) instrument is then used to pattern nanochannels in the substrate to connect the two microchannels. Each nanochannel has three asymmetrically spaced 50 × $50 \mathrm{~nm}$ nanopores. To mill nanochannels directly into glass substrates, we use an electron flood gun to compensate for charge buildup from the ion beam. FIB milling permits the fabrication of a wide range of sensing geometries, as well as the direct characterization of the patterned substrates with the integrated scanning electron microscope (SEM). The micro- and nanochannels are sealed by thermally annealing a cover plate to the substrate. A schematic of the device is presented in Figure 1a.

In the resistive-pulse sensing experiments, $\mathrm{HBV}$ capsids in a buffered $1 \mathrm{M} \mathrm{NaCl}$ solution are placed in the capsids reservoir (Figure 1a). A positive potential of $200-400 \mathrm{mV}$ is applied across the capsids and waste reservoirs and electrokinetically drives the capsids through the bridging nanochannel, where a change in current is generated as capsids transit each pore. Figure $2 \mathrm{a}$ shows the variation in current with time through the nanochannel, for two applied potentials. Each current pulse corresponds to the passage of one capsid through one nanopore, and the pulse amplitude is proportional to the capsid volume. As the applied potential increases, pulse amplitude increases, and pulse width decreases. Accordingly, the transit time between pores also decreases, as the capsids velocity increases. Interestingly, these trends are observed until a threshold potential of $>300 \mathrm{mV}$ is reached. As shown in the plot of pulse width with potential in Figure $2 \mathrm{~b}$, the pulse width increases dramatically at high potentials. The longer pulse widths may be explained by DC dielectrophoresis [2], whereby the virus capsids become trapped in the nanopores by the high field gradients. Below the threshold potential, however, electrophoretic transport is still the dominant force acting on the particles. Further characterization of the balance of forces acting upon the capsids will allow an optimized sensing scheme to be used in the biological experiments [3]. 


\section{References:}

[1] Z. Harms et al, Analytical Chemistry 83 (2011), p. 9573-9578.

[2] F. Camacho-Alanis, L. Gan, and A. Ros, Sensors and Actuators B 173 (2012), p. 668-675.

[3] This work was supported in part by NSF CHE-0832651, NSF CHE-0923064, and NIH R01 GM100071.
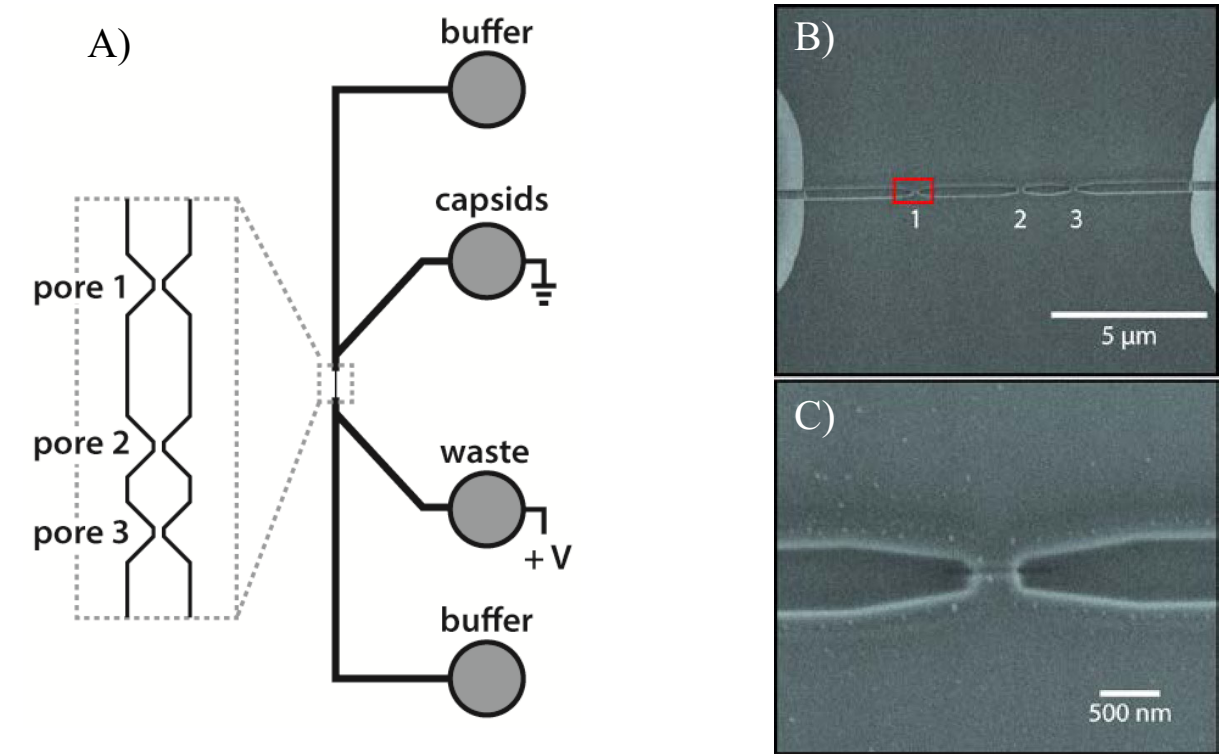

Figure 1. a) Schematic of the microchannel-nanochannel device. b) SEM micrograph of the three pores in series (c) and a magnified view of pore one from (b).
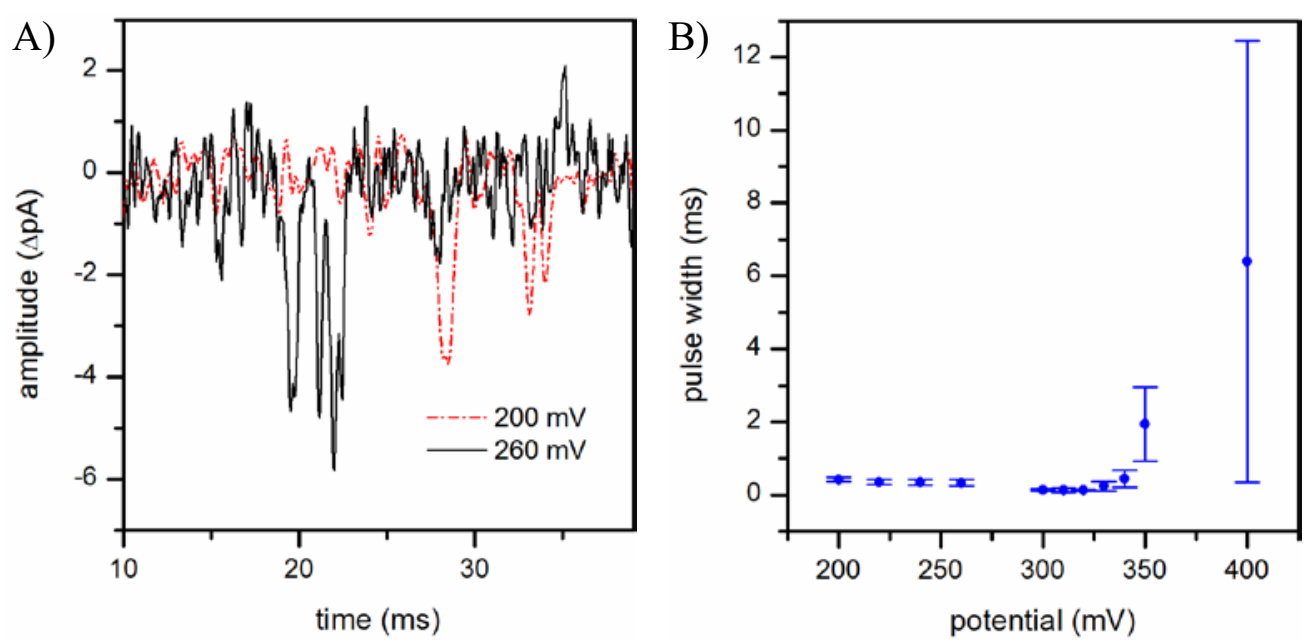

Figure 2. a) Resistive-pulse sensing events corresponding to the transit of two capsids through three pores at two different potentials. At low potentials, the pulse width and the transit time between pores decrease with increasing electric field strength. $b$ ) The width of pulse one (corresponding to pore one in Figure 1c) is plotted as a function of potential across the device. Pulse width decreases until a threshold potential of $325 \mathrm{mV}$ is reached, from which point the width increases. 